\title{
Evolution of Quantum Teleportation Circuits with Improved Genetic Algorithm
}

\author{
Swanti Satsangi \\ Department of Physics and Computer Science \\ Dayalbagh Educational Institute \\ Agra, India
}

\author{
C. Patvardhan \\ Department of Electrical Engineering \\ Dayalbagh Educational Institute \\ Agra, India
}

\begin{abstract}
Quantum Teleportation is the process of transmitting Quantum information from one place to another by performing Quantum mechanical operations like entanglement, Bell measurement and classical communication. Unlike the commonly used term teleportation, Quantum teleportation involves only transfer of information rather than particles or objects.
\end{abstract}

Quantum teleportation allows transmission of quantum information despite the impossibility of measuring or broadcasting the information to be transmitted.

This paper evolves some new simpler Quantum teleportation circuits which use only Quantum entanglement and a set of Hadamard and CNOT gates. Further, each of the circuits performs teleportation even if Alice does not perform measurement on her qubits. Keeping the feasibility of physical realization in mind, circuits using only adjacent CNOT gates along with Hadamard gates have also been constructed. All these circuits have been evolved using Genetic Algorithms which has been designed with appropriate representation and search operations towards faster convergence.

\section{Keywords}

Quantum teleportation, Quantum circuit, Entanglement, Adjacent gates, Genetic algorithm.

\section{INTRODUCTION}

Quantum computation is a flourishing research area and quantum computers are believed to hold immense computational advantage over classical ones [18]. This rapidly evolving field is one of the most active research areas of modern science. The primary reason for the growing interest in the field is that the ever shrinking size of the integrated circuits in present day computers has led to atomic scale computing and in the coming years, the current technology is expected to encounter a dead end. This is expected to lead to reliance on quantum mechanical phenomena for computing and building Quantum computing devices.

A quantum computer is a device for computation that makes direct use of quantum mechanical phenomena, such as superposition and entanglement, to perform operations on data. The basic principle behind quantum computation is that quantum properties can be used to represent data and perform operations on this data. Although scientists are not sure what type of quantum computing devices will ever be possible to build, it is expected that quantum computers will be able to solve some interesting problems more efficiently than any known classical counterparts, such as integer factorization, discrete logarithms, and quantum simulation and modeling that are intractable on any present or future conventional computer.
A mysterious quantum phenomena, Quantum Teleportation, is expected to play an important role in quantum computing technology. It was first suggested by Bennett et al. [3] and is considered to be one of the most remarkable milestones of Quantum computation and Quantum communication. Apart from other operations on qubits to transfer quantum information between two distant parties, Teleportation utilizes one of the most mysterious aspects of Quantum computing, i.e. entanglement. Albert Einstein did not believe in Quantum Mechanics since he believed that "physics should represent a reality in time and space, free from spooky actions at a distance"[9]. This spooky action was nothing but Quantum entanglement which is a quantum phenomenon unifying two distant particles in such a way that an operation on one of the particles instantly affects the other, irrespective of the physical distance between them.

Gottesman and Chuang claimed that a generalization of quantum teleportation can lead to construction of universal quantum computers [11]. They have shown that a single technique i.e. teleportation, is enough to construct a fault tolerant quantum computer using single qubit operations, Bell measurement and a few entangled quantum states like GHZ (Geeenberger-Home-Zeilinger) states. Since, all these operations are already realizable with the existing technology, it is possible to build a universal quantum computer with the help of teleportation.

Teleportation has received great attention since its pioneer proposal in 1999, mainly after its experimental realizations. The crucial ingredient characterizing this phenomenon is the transfer of information between non interacting systems, at the expense of a quantum channel. According to Brassard [6], in terms of technological implementation, teleportation is the most difficult problem when compared to Quantum Cryptography, number factoring or any other problem in Quantum computing. This is due to the fact that teleportation requires to be performed as soon as the Quantum states are generated since long-term storage of quantum information is very difficult. On the other hand, Brassard also claimed that the circuit implementation of Quantum teleportation is much easier than any other Quantum computational task

Since teleportation holds such tremendous power, it has always been an area of interest for researchers. In this view, this paper attempts at designing new Quantum teleportation circuits that are simpler than the existing circuits in terms of the gate library required for their construction and the number of gates required to build them. The paper has been divided into various sections. Section 2 briefly discusses the concept of teleportation, it's probable applications, earlier attempts at designing teleportation circuits and its various practical implementations done by research groups across the globe. Section 3 describes in detail, the algorithm and its steps that have been adopted in order to evolve the teleportation circuits. Section 4 discusses the various circuits which have been 
evolved here and section 5 finally summarizes the study.

\section{TELEPORTATION}

Teleportation of a Quantum state $|\psi\rangle$ from point A to another point B, usually termed as Alice and Bob respectively, involves the following steps.

1. Entanglement of qubit states owned by Alice and Bob.

2. Preparation of teleportee state $|\psi\rangle$

3. Entanglement of Alice's qubit with $|\psi\rangle$

4. Bell measurement on two qubits possessed by Alice

5. Communication of Alice's measurement result to Bob by classical channel and

6. Operation on Bob's qubit depending on Alice's measurement results.

The process can be best understood with the following circuit given by Bennett et al. [3]

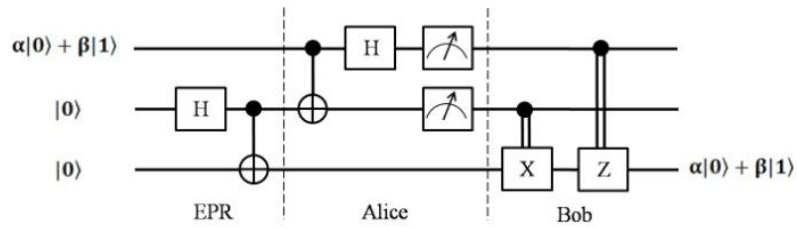

Figure 1. Teleportation circuit proposed by Bennett et al.

\subsection{Applications of Teleportation}

Quantum cryptography, specifically Quantum key distribution is one important field where quantum teleportation can be practically applied to provide completely secure information exchange between two distant communicators without the interference of an eavesdropper making it impossible to intercept without the classical channel[2].

Apart from cryptography, as suggested by Gottesman et al., teleportation can be implemented to construct a universal quantum computer[11].

Yamanoue et al. [29] suggested that computer simulation of teleportation can be applied in 3D modeling and self reproduction by extracting all the relevant information from an object and sending it to a remote location.

The most awaited application of quantum teleportation would be the teleportation of humans from one place to another. But scientists claim that this might not be possible in the near future as it would involve storage, transfer and replication of information of trillions of atoms in human body from one place and then reconstructing identical information at another and till date, teleportation of only single qubit has been experimentally realized [3]

\subsection{Previous Attempts at Designing Teleportation Circuits}

Quantum teleportation is one of the quintessential quantum phenomena and efforts have been reported in the literature for designing a variety of teleportation circuits. While the first computational circuit given by Brassard was hand crafted [6], a few other researchers utilized evolutionary algorithms for the purpose of designing their circuits. Evolutionary algorithms are search and optimization algorithms that form a colony of individuals and optimize the given problem by finding the individual with the highest fitness [14]. These algorithms require minimal prior knowledge about the problem or the search space and therefore find a wide variety of applications [8].

Williams and Gray were the first ones to adopt genetic programming for evolution of teleportation circuit [27]. Application of GA for evolving teleportation circuit has also been reported by Yabuki et al. [28]. They successfully evolved a teleportation circuit using only eight gates while the circuits proposed earlier by Brassard [6] and Williams et al. [27] were composed of eleven and nine gates respectively. The circuit obtained by them have been given below.

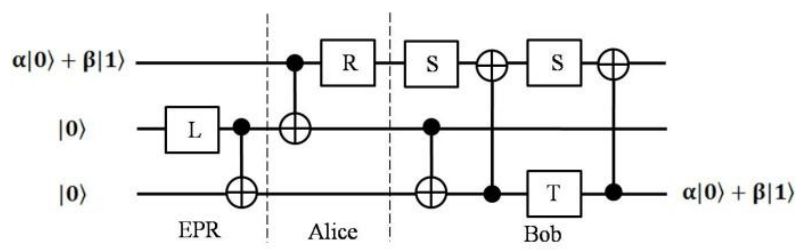

Figure 2. Teleportation circuit designed by Brassard

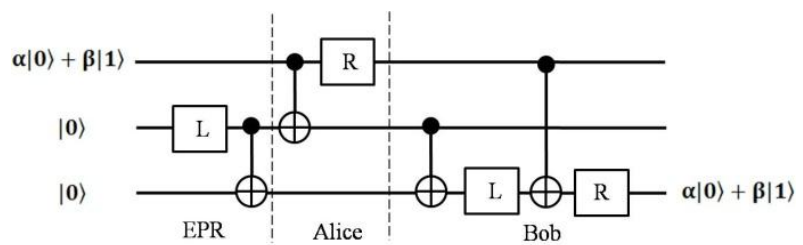

Figure 3. Teleportation circuit designed by Williams et al.

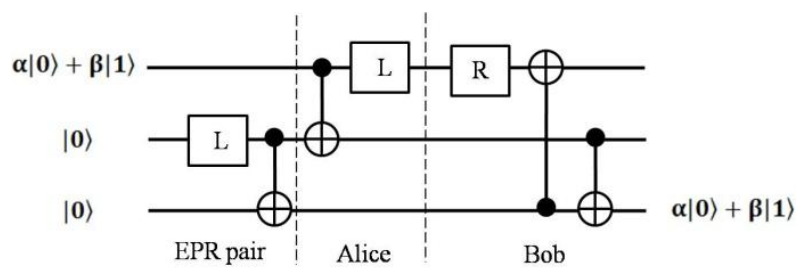

Figure 4 Teleportation circuit designed by Yabuki et al.

\subsection{Practical Demonstrations of Teleportation}

Since 1993, when the concept was first introduced by Bennett et al., numerous attempt have been made to practically implement Quantum teleportation. Quantum teleportation was first demonstrated with entangled photons [5] in 1997. Various developments have been achieved in laboratory, including the demonstration of entanglement swapping [19], open destination teleportation [31] and teleportation of two bit composite system [30]. Entanglement distribution has been shown with fiber links[15]. In addition, "practical" quantum teleportation have been realized via fiber links [19] and limited to a distance of about one kilometer. Experiments have achieved free space distribution of entangled photon pairs over distances of $600 \mathrm{~m} \mathrm{[1]} \mathrm{and} 13 \mathrm{~km}$ [20]. Later, entangled photons were transmitted over $144 \mathrm{~km}$ [25]. In these experiments, either only one photon was transmitted [10] or the entangled photon pair was transferred together using only a one-link channel. Most recently, following a modified scheme[4], quantum teleportation over a $16 \mathrm{~km}$ freespace links was demonstrated [13] with a single pair of entangled photons. 


\section{DESIGNING SIMPLER TELEPORTATION CIRCUITS}

As mentioned earlier, Brassard in the first place, proposed a teleportation circuit with $\mathrm{L}, \mathrm{R}, \mathrm{S}$ and $\mathrm{T}$ gates along with CNOT while Yabuki et al. [28], similar to Williams et al. [27], included L, R and CNOT gates apart from measurement in their circuit, where $L=\frac{1}{\sqrt{2}}\left[\begin{array}{rr}1 & -1 \\ 1 & 1\end{array}\right], R=\frac{1}{\sqrt{2}}\left[\begin{array}{rr}1 & 1 \\ -1 & 1\end{array}\right]$, $S=\left[\begin{array}{ll}i & 0 \\ 0 & 1\end{array}\right], T=\left[\begin{array}{rr}-1 & 0 \\ 0 & -i\end{array}\right]$ and CNOT gate works like a classical XOR gate which flips the target bit only if the controlling bit is 1 . In this paper, only Hadamard gate defined as $H=\frac{1}{\sqrt{2}}\left[\begin{array}{rr}1 & 1 \\ 1 & -1\end{array}\right]$, has been utilized other than CNOT gate, to obtain teleportation circuits.

The circuits designed in this work are simpler than the existing ones due to the fact that the gate library used here is smaller than the earlier works. Further, the gates used are universal quantum gates that can be used to perform any possible operation on a quantum computer[27]. A comparative table of gate libraries has been given below.

Table 1. Gate libraries used in various earlier researches

\begin{tabular}{|c|c|c|}
\hline S. no. & Reference & Gate library used \\
\hline 1. & Brassard [6] & L, R, S, T, CNOT \\
\hline 2. & Williams et al. [27] & L, R, CNOT \\
\hline 3. & Yabuki et al. [28] & L, R, CNOT \\
\hline 4. & Proposed Approach & H, CNOT \\
\hline
\end{tabular}

\subsection{Proposed GA-based approach for evolving teleportation circuit}

For the purpose of evolution of a teleportation circuit, Genetic algorithm has been employed in this work. Genetic algorithms are a class of evolutionary algorithms which utilize the Darwinian principle of the survival of the fittest, along with features like selection, crossover and mutation, in order to obtain an optimum solution for a given problem. The algorithms has been described in detail below.

\subsubsection{Gate representation}

The gate library is divided into three sets, one each for EPR pair generation, Alice's operation and Bob's retrieval circuit. Each set comprises of the two gates operating on different qubits, symbolically represented in $\mathrm{GATE}_{\text {qubit }}$ format. So the symbol $\mathrm{H} 1$, in tables 1 and 3, represents a Hadamard gate operating on qubit 1 . Similarly, $\mathrm{CNOT}_{1,2}$ is a CNOT gate with qubit 1 as control and qubit 2 as target. Integer representation has been chosen here as a pointer to each gate i.e. each gate is assigned a unique integer such that the same gate operating on different qubits is denoted by a different integer. This representation is simpler than the one adopted by Yabuki et al. where a set of three integers represented a gate [28]. Apart from integer, binary, symbolic and real representation can also be used [21]

Table 2. Gate library for EPR pair generation

\begin{tabular}{|c|c|}
\hline Gate & Number \\
\hline $\mathrm{H}_{1}$ & 1 \\
\hline $\mathrm{H}_{2}$ & 2 \\
\hline $\mathrm{CNOT}_{1,2}$ & 3 \\
\hline $\mathrm{CNOT}_{2,1}$ & 4 \\
\hline
\end{tabular}

Table 3. Gate library for Alice's operation

\begin{tabular}{|c|c|}
\hline Gate & Number \\
\hline $\mathrm{H}_{2}$ & 1 \\
\hline $\mathrm{H}_{3}$ & 2 \\
\hline $\mathrm{CNOT}_{2,3}$ & 3 \\
\hline $\mathrm{CNOT}_{3,2}$ & 4 \\
\hline
\end{tabular}

Table 4. Gate library for Bob's qubit retrieval circuit

\begin{tabular}{|c|c|}
\hline Gate & Number \\
\hline $\mathrm{H}_{1}$ & 1 \\
\hline $\mathrm{H}_{2}$ & 2 \\
\hline $\mathrm{H}_{3}$ & 3 \\
\hline $\mathrm{CNOT}_{1,2}$ & 4 \\
\hline $\mathrm{CNOT}_{1,3}$ & 5 \\
\hline $\mathrm{CNOT}_{2,1}$ & 6 \\
\hline $\mathrm{CNOT}_{2,3}$ & 7 \\
\hline $\mathrm{CNOT}_{3,1}$ & 8 \\
\hline $\mathrm{CNOT}_{3,2}$ & 9 \\
\hline
\end{tabular}

\subsubsection{Population generation}

A population with $n$ chromosomes is initialized randomly. Size of each chromosome is fixed to length $m$, where $m=$ $m_{1}+m_{2}+m_{3}$ and $m_{1}, m_{2}, m_{3}$ are the number of gates in EPR pair generation circuit, Alice's circuit and Bob's circuit respectively. Thus each chromosome is nothing but a string of integers representing a quantum circuit with $m_{1}$ gates from EPR library, $m_{2}$ gates from Alice's library and $m_{3}$ gates from Bob's library. Since gene representation is in the form of integers and not codons as in the work by Yabuki et al. [28], population representation becomes much more simpler to code and decode. Another possible representation of the population could be a second order representation as suggested by Massey et al. but this again becomes complex since high level execution is required to decode and obtain the circuits [16].

Consider an example of a population with 4 chromosomes; each consisting of 8 gates, with $m_{1}=m_{2}=2$ and $m_{3}=$ 4. Then a possible combination of the population could be written as:

$$
\text { Pop }=\left\{\begin{array}{llllllll}
1 & 2 & 2 & 3 & 4 & 3 & 3 & 5 \\
3 & 4 & 3 & 1 & 3 & 8 & 9 & 1 \\
4 & 3 & 1 & 2 & 3 & 9 & 2 & 7 \\
1 & 3 & 2 & 3 & 5 & 1 & 4 & 6
\end{array}\right\}
$$

Figure 5. Example of initial population

\subsubsection{Circuit Construction}

Once the population of circuits is generated, the sequence of integers is decoded into a sequence of gates and then the unitary transformation matrix is calculated for each chromosome using a series of Kronecker products and matrix multiplications. The serial connection of quantum gates 
corresponds to the standard matrix multiplication of the unitary matrices representing the gates, and the parallel connection corresponds to Kronecker multiplication of the unitary matrices. For instance, consider a chromosome $\left[\mathrm{H}_{1} \mathrm{CNOT}_{21} \mathrm{H}_{3} \mathrm{CNOT}_{23} \mathrm{CNOT}_{12} \mathrm{CNOT}_{32} \mathrm{H}_{2}\right]$, then the unitary matrix for this circuit will be calculated as follows:

Step 1 = Kronecker(I, Kronecker $(\mathrm{I}, \mathrm{H}))$

Step $2=$ Kronecker(I, CNOT $\left._{21}\right)$

Step 3 = Kronecker(H, Kronecker(I, I))

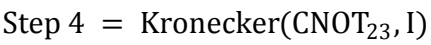

Step $5=$ Kronecker $\left(\mathrm{I}, \mathrm{CNOT}_{12}\right)$

Step $6=$ Kronecker $\left(\mathrm{CNOT}_{32}, \mathrm{I}\right)$

Step 7 = Kronecker(I, Kronecker(H, I))

Circuitmatri $=$ Step7 $*$ Step $6 *$ Step $5 *$ Step $4 *$ Step3

$$
\text { * Step } 2 * \text { Step } 1
$$

\subsubsection{Evaluation}

Evaluation comprises of three steps:

- Calculation of fitness of population

- Selection of parents for reproduction

- Crossover and mutation

\subsubsection{Fitness function}

Matrix based approach for fitness assessment has been implemented in this work [22]. This technique was in the first place proposed by Williams et al. [27]. A pre-condition for the approach is that the desired circuit matrix should be known.

A target unitary matrix of a circuit already performing teleportation is given as input. The calculated circuit matrix is compared component wise and a difference matrix is obtained. The difference matrix is a binary matrix with the value 1 whenever an element of obtained matrix is same as the desired matrix, and 0 otherwise. Thus, the fitness is calculated as:

$$
\text { Fitness }=\frac{\text { No. ofmatching element }}{\text { Total elements in difference matrix }}
$$

The algorithm targets to obtain a perfect fitness of 1 .

\subsubsection{Selection}

If the desired fitness is not obtained, a set of reproducing parents is selected using fitness-proportionate selection technique called the Roulette Wheel Selection. Based on the fitness level of the chromosome, a slice of the roulette wheel is allotted to it and the wheel is spun as many times as the number of parents is required. These parents then form a pool for crossover and mutation. In the process one or more individuals, usually the fitter ones, maybe selected multiple times since they have a bigger share on the wheel than their weaker rival.

\subsubsection{Crossover and Mutation operators}

Once the parents are selected, two-point crossover and mutation are performed separately for all three sections of the circuit, i.e. EPR circuit, Alice's circuit and Bob's circuit. After crossover and mutation, these separate strings are recombined into a new population which are re-evaluated for their fitness as explained in previous section.

The process of circuit calculation and evaluation is repeated with the updated population till the circuit with desired fitness is not obtained.

\section{EXPERIMENTS AND RESULTS}

Two sets of experiments were performed using the following input parameters:

Size of population : 20

No. of input qubits : 3

Max. no. of gates: 8

Probability of crossover: .7

Probability of mutation: .1

Two different gate libraries were constructed; one with all possible CNOT gate combinations and another with CNOT gate acting only on adjacent qubits, along with Hadamard gate.

A separate gate library with only adjacent gates was developed keeping in mind the feasibility of physical realization in mind of the circuits in mind. It has been suggested by researches that the linear nearest neighbor architecture is preferable for quantum information processing [17].

A circuit giving the desired output was obtained in each run of the algorithm and the time taken in each run varied from 2 40 seconds depending on the size of population. Population size was selected after a series of experiments with different sizes. It was observed that size of population had a direct effect on the evolution time. Evolution time was minimum for a population of 20 chromosomes and increased with the increase in population size. This can be seen in the graph below.

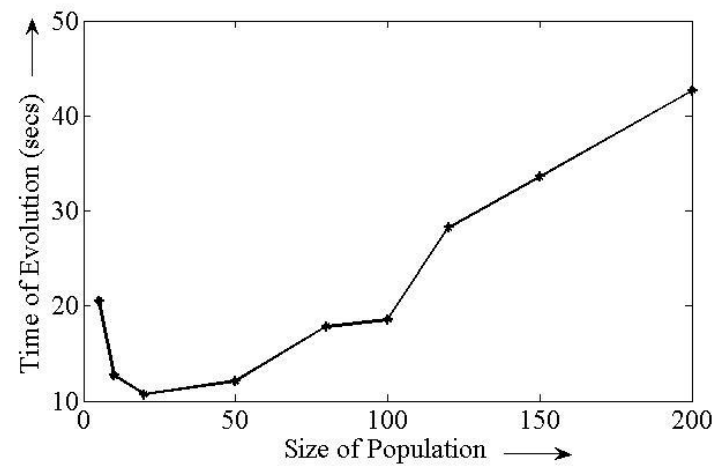

Figure 6. Effect of Population Size on Evolution Time

The obtained results have been divided into three categories discussed below.

\subsection{Teleportation circuits using all gate combinations}

The circuits evolved using the above discussed algorithm have been shown in figure 7 and figure 8 , where figure 7 shows the seven gate teleportation circuit and figure 8 show the eight gate teleportation circuit. Both the circuits give identical superposition states on the top two wires, which can be determined by measurement, and the teleported state is obtained on the third wire.

Thus, for any initial state $|\Psi\rangle=|\psi\rangle|0\rangle|0\rangle$, the circuits transfer the state $|\psi\rangle=\alpha|0\rangle+\beta|1\rangle$ from top wire to bottom wire. 


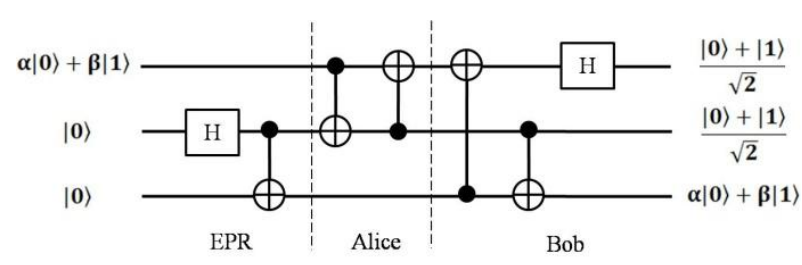

Figure 7. Evolved teleportation circuits using seven gates

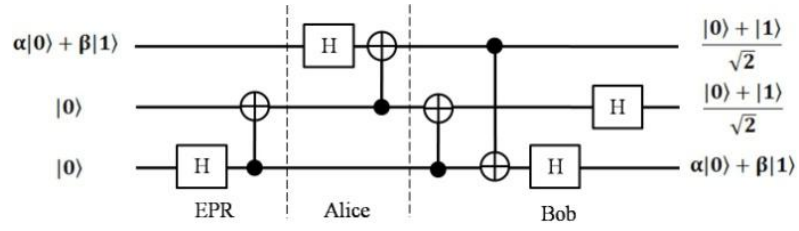

Figure 8. Evolved teleportation circuits using eight gates

The obtained circuits have been compared to the existing ones in terms of their gate composition (measurement excluded) in table 5 .

Table 5. Comparison of the obtained circuits with the existing

\begin{tabular}{|c|c|c|c|c|}
\hline S. No. & Reference & $\begin{array}{c}\text { Total } \\
\text { No. } \\
\text { Of } \\
\text { Gates }\end{array}$ & $\begin{array}{c}\text { No. Of } \\
\text { Single } \\
\text { Qubit } \\
\text { Gates }\end{array}$ & $\begin{array}{c}\text { No. Of } \\
\text { Two } \\
\text { Qubit } \\
\text { Gates }\end{array}$ \\
\hline 1 & Brassard [3] & 10 & 5 & 5 \\
\hline 2 & Williams et al. [27] & 8 & 4 & 4 \\
\hline 3 & Yabuki et al.[28] & 7 & 3 & 4 \\
\hline 4 & Proposed Approach & 7 & 2 & 5 \\
\hline
\end{tabular}

\subsection{Teleportation circuit using only adjacent gate combinations}

With only adjacent CNOT gates with Hadamard gates, a teleportation circuit with a total of 8 gates has been evolved using the algorithm explained in the previous section. The circuit is given below.

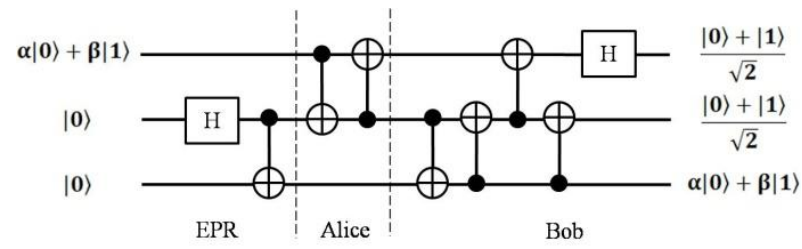

Figure 9. Teleportation circuit using only adjacent gates

\subsection{Other Circuits}

During the evolution process, a few other circuits were evolved. Although these circuits had a perfect fitness of 1 , transferred the input qubit from top wire to bottom and had fewer gates than existing circuits, they were incorrect. This is because in the first two circuits in figure 10 and figure 11, Alice must entangle her qubit with the unknown qubit but it was found that in the given circuits, Alice's qubit is only entangled to Bob's. The third circuit in figure 12 also gives the desired result but it is simply a copy circuit and not a teleportation circuit. Thus despite transferring the unknown state from qubit 3 to qubit 1 , the three circuits are not valid teleportation circuits.

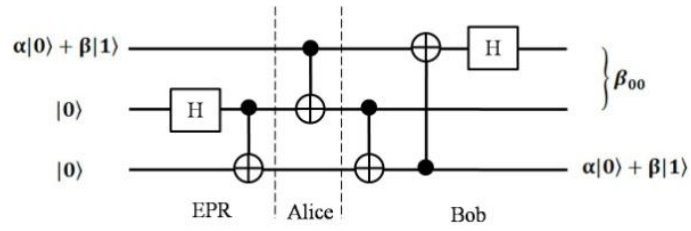

Figure 10. Incorrect circuit 1

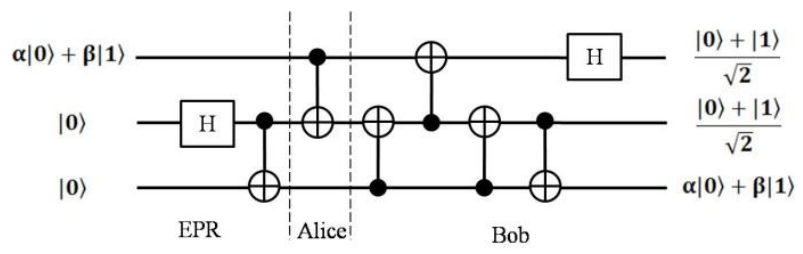

Figure 11. Incorrect circuit 2

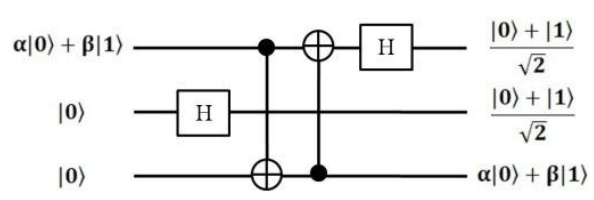

Figure 12. Incorrect circuit 3

\section{CONCLUSIONS}

The paper addresses design of simpler teleportation circuits in the field of quantum computing. Genetic algorithm has been applied to evolve a variety of Quantum teleportation circuits which are simpler than any of the reported circuits till date. It has also been shown that teleportation can be performed with as few as 7 gates, which is smaller than the best known circuit. Further, while several physical realization schemes have been proposed for future quantum information processing, most known facts suggest that quantum information processing should have intrinsic limitations; physically realizable operations would be only interaction between neighbor qubits. Most of the existing researches do not consider changing the gate order of the original circuit, and thus the result may not be optimal. In view of challenges in practical implementations of quantum gates and circuits, a circuit using only adjacent gates has also been shown.

\section{REFERENCES}

[1] Aspelmeyer M. Böhm H. R., Gyatso T., Jennewein T., Kaltenbaek R., Lindenthal M., Terriza G. M., Poppe P., Resch K., Taraba M., Ursin R., Walther P., Zeilinger A., 2003, Long-distance free-space distribution of quantum entanglement,Science, 301, 621.

[2] Bashar M. A., Chowdhury M. A., Islam R., Rahman M. S., Das S. K., 2009, A Review and Prospects of Quantum Teleportation, MASAUM Journal of Basic and Applied Sciences Vol.1, No. 2.

[3] Bennett, C. H., Brassard G., Cr'epeau C., Jozsa R., Peres A. and Wootters W., 1993, Teleporting an unknown quantum state via dual classical and Einstein-PodolskyRosen channels, Physical Review Letters 70:13, 1895 1899.

[4] Boschi D. Branca S., Martini F. De, Hardy L., and Popescu S., 1998, Experimental Realization of Teleporting an Unknown Pure Quantum State via Dual Classical and Einstein-Podolsky-Rosen Channels, Physical Review Letter 80, 1121.

[5] Bouwmeester D., Pan J. W, Mattle K., Eibl M., Weinfurter H. and Zielinger A., 1997, Experimental 
Quantum Teleportation, Nature 390, 575.

[6] Brassard G., 1996, Teleportation as a quantum computation. In Proceedings of the Fourth Workshop on Physics and Computation, page 44. New England Complex Systems Institute, quantph/ 9605035.

[7] Buchler B., 2013, Teleportation just got easier-but not for you, unfortunately, The Conversation, Quantum Physics, Physics.org,

[8] Coello C.A.C., 2005, An introduction to evolutionary algorithms and their applications. In: Corchado FFR, Larios-Rosillo V, Unger H (eds) ISSADS, vol 3563 of Lecture Notes in Computer Science. Springer, Berlin, pp 425-442.

[9] Einstein, A., Podolsky, B. and Rosen, N., 1935, Can quantum mechanical description of physical reality be considered complete?, Phys. Rev., 47, 777-780.

[10] Fedrizzi, A. et al., 2009 High-fidelity transmission of entanglement over a high-loss free-space channel. Nature Phys. 5, 389-392.

[11] Gottesman D. and Chuang I. L., 1999, Demonstrating the viability of universal quantum computation using teleportation and single-qubit operations, Nature, Vol. 402, 25 November.

[12] International Business Machines, February 1996, Stand by: I'll teleport you some goulash, Scientific American.

[13] Jin X. M.,Ren J. G., Yang B., Yi Z. H., Zhou F., Xu X. F., 2010, Experimental Free-space Quantum Teleportation, Nature Photonics 4, 376.

[14] Koza J. R. 1992, Genetic programming: on the programming of computers by means of natural selection. MIT Press, Cambridge.

[15] Marcikic I., Riedmatten H. De, Tittel W., Zbinden H., Legré M., and Gisin N., 2004, Distribution of Time-Bin Entangled Qubits over $50 \mathrm{~km}$ of Optical Fiber, Physical Review Letter 93, 180502.

[16] Massey P., Clark J. A., and Stepney S., 2005, Evolution of a human-competitive quantum fourier transform algorithm using genetic programming. In: Proceedings of the 2005 conference on genetic and evolutionary computation, ACM Press, New York, pp 1657-1663.

[17] Matsuo A. and Yamashita S., Changing the Gate Order for Optimal LNN Conversion, Reversible Computtion, LNCS, Vol. 7165, Pp. 89-101.

[18] Nielson M. A. and Chuang I. L. 2000, Quantum Computing and Quantum Information. Cambridge University Press.

[19] Pan J. W., Bouwmeester D. ,Weinfurter H. and Zeilinger
A., 1998, Experimental entanglement swapping: entangling photons that never interacted. Phys. Rev. Lett. Vol. 80, 3891.

[20] Peng C. Z. et al., 2005, Experimental free-space distribution of entangled photon pairs over $13 \mathrm{~km}$ : towards satellite-based global quantum communication, Physcal Review. letter 94, 150501.

[21] Rothlauf F., 2006, Representations for genetic and evolutionary algorithms, 2nd edn. Springer, Berlin.

[22] Satsangi S., Gulati A., Kalra P. K, and Patvardhan C., 2012, "Application of Genetic Algorithms for Evolution of Quantum Equivalents of Boolean Circuits", International Journal of Electrical, Computer, Electronics and Communication Engineering, Vol. 6, No. 3.

[23] Satsangi S. and Patvardhan C., 2015, Application of Genetic Algorithm for Evolution of Quantum Fourier Transform Circuits, Proceedings of the Second International Conference on Computer and Communication Technologies, Vol. 379, pp. 773-782.

[24] Ursin, R., Jennewein T., Aspellmeyer M., Kaltenbaek R., Lindenthal M., Walthar P. and Zelinger A. 2004, Quantum teleportation across the Danube. Nature 430, 849.

[25] Ursin R., Tiefenbacher F., Schmitt M., Weier H., Scheidl T., Lindenthal M., Blauensteiner B., Jennewein T., Perdigues J.,Trojek P., Ömer B., Fürst M., Meyenburg M., Rarity J., Sodnik Z., Barbieri C., Weinfurter H. and Zeilinger A., 2007, Entanglementbased quantum communication over $144 \mathrm{~km}$, Nature Physics 3, 481 .

[26] Wikipedia,https://en.wikipedia.org/wiki/Quantum_gate\# Universal_quantum_gates

[27] Williams C. P. and Gray A. G., 1999, Automated design of quantum circuits. In QCQC'98 LNCS 1509, Pp. 113. Springer-Verlag.

[28] Yabuki T and Iba H., 2000, Genetic algorithms for quantum circuit design - Evolving a simpler teleportation circuit. In Late Breaking Papers at GECCO,

[29] Yamanoue T., Tsutsui Y. and Tsutsui T., 2002, Realizing a Practical Teleportation System Using the Intelligent Parts, International Conference On Information Technology \& Applications, 25-28.

[30] Zhang Q., 2006, Experimental quantum teleportation of a two-qubit composite system, Nature Physics 2, 678.

[31] Zhao Z., Chen Y. A, Zhang A. N, Yang T., Briegel H. J. and Pan J. W, 2004, Experimental demonstration of fivephoton entanglement and open-destination teleportation, Nature 430, 54. 